\title{
The Effect of Film Temperature on crater morphology during the impact of a single droplet
}

\author{
D. B. Hann, E.R. Underhill and A. Mitchell \\ Faculty of Engineering, university of Nottingham, UK
}

\begin{abstract}
The effect of varying the film temperature from $15 \mathrm{C}$ to $60 \mathrm{C}$ is investigated using high speed video imaging. It is demonstrated that increasing the temperature difference between the droplet and film liquid changes the size and frequency of the secondary droplets. The impact can be split into three regimes. In the crater expansion regime, the crater follows a self-similar behaviour. In the second stage, the crater becomes deeper and wider at higher temperatures possibly due to decrease in the viscosity or surface tension. It is seen that the crater collapse is less dependent on the temperature and occurs at fixed time for a particular Webber number.
\end{abstract}

\section{Introduction}

The phenomenon of droplets impacting onto both liquid and solid surfaces has been of great interest since the early 1900s, one of the first to investigate the characteristics of splashing being [1]. The interest in droplet impact has stemmed from its appearance in many engineering applications, a few examples listed by [2] include; ink-jet printing, spray cooling of turbine blades and internal combustion engines. Within internal combustion engines the lubricating oil is sprayed from the rotating parts onto the interior walls, quickly creating an oil film. The following oil droplets will then inevitably splash when impacting onto the film, creating secondary drops. Minimising these secondary drops is of great importance to engine manufacturers as they are believed to reduce efficiency. This study is therefore relevant to industry by developing an understanding to how temperature may affect the outcome of droplet impact onto liquid films.

There are several reviews of the droplet impact outcomes, such as [3], and there are a number of theories about the crater evolution [4] for droplets of same temperature and liquid impacting on a film [5]. However there are few studies on droplets of different temperature to the film $[6,7]$. This work is part of an experimental study to provide data for comparison with models to confirm or deny if they are still valid in the case where the film liquid has different properties.

\section{Experimental methodology}

The apparatus for the experiment is shown in figure 1 . The control variables that were kept constant throughout the experiment were; the droplet diameter and the film thickness. The independent variables were; the impact velocity and the film temperature, and the dependent variables were the crater dimensions and the splash regimes. 
Five different film temperatures were investigated; $15^{\circ} \mathrm{C}, 30^{\circ} \mathrm{C}, 40^{\circ} \mathrm{C}, 50^{\circ} \mathrm{C}$ and $60^{\circ} \mathrm{C}$. At each temperature five different initial heights of the primary droplet were used in increments of $50 \mathrm{~mm}$ from $100 \mathrm{~mm}$ to $300 \mathrm{~mm}$. Each droplet was repeated five times in order to demonstrate the repeatability and the reliability of the results. In total there were 125 droplets analysed.

The videos from the high-speed camera were analysed using MATLAB software similar to that of the work from [5]. Any measurements from the images were originally taken in pixels and then calibrated to $\mathrm{mm}$. The precision was 1 pixel which translated on average to $0.065 \mathrm{~mm}$. Each droplet size and velocity was individually measured to eliminate errors from movement of the camera or film between tests. The dimensions as well as time ( $\mathrm{t}$ ) were non-dimensionalised for comparison with the work from [8] and [4]using the following relationships.

$$
z^{*}=\frac{Z}{D}, \Omega=\frac{w}{D}, \tau=\frac{t U}{D}
$$

Where; $\mathrm{z}$ is the crater depth, $\mathrm{w}$ is the crater width, $\mathrm{t}$ is the time after impact, $\mathrm{D}$ is the droplet diameter

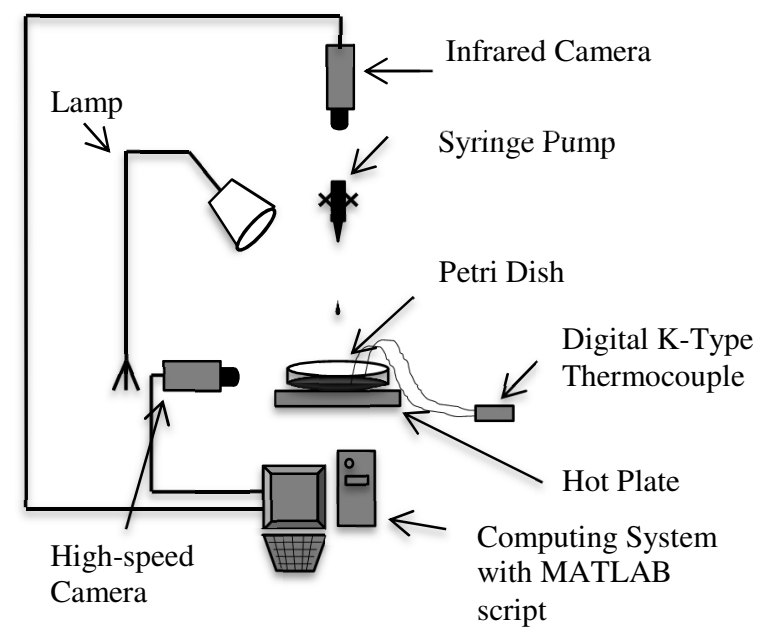

Figure 1: Diagram of experimental set up used

and $\mathrm{U}$ is the droplet velocity measured just before the impact.

\section{Results}

The effect of the film temperature on the impact can be easily determined in the sequences of images shown in figure (2). At $50 \mathrm{C}$ it is clear that the secondary droplets generated from the jet formed after collapse of the impact crater are smaller and more frequent. This might be expected since the surface tension and viscosity of the film liquid are decreased by an increase in temperature.

For each of the five sequences of droplet impact parameters, the location of the base of the impact crater and the width of the crater can be determined from the Matlab code. These were averaged and it was seen that the impact was highly repeatable with little variation between each of the repeats. The average of the crater depth and width are shown in figure 3. In both cases it can be seen that in the inertial self-similar region $\left(\tau=\frac{t U}{D}<5\right.$ ) is not affected by the variation in the film temperature. The effect of the film temperature becomes evident during the collapse of the impact crater which is dominated by the Weber number. When the film temperature increases, the crater becomes deeper and wider before it starts to collapse in response to the lower surface tension and viscosity. There is an indication that the time for crater collapse is the same for all temperatures. 


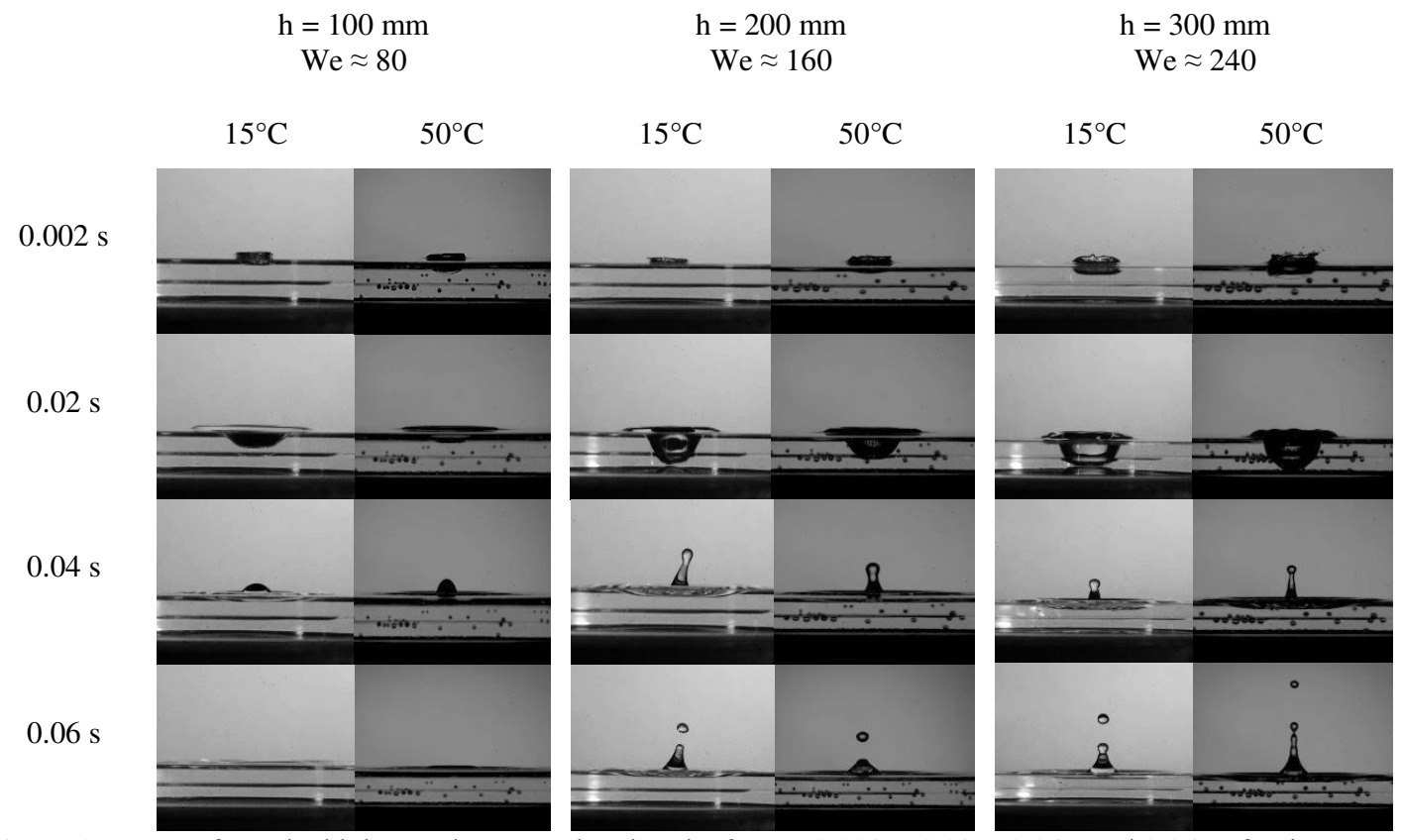

Figure 2: Images from the high-speed camera showing the frames $0.002 \mathrm{~s}, 0.02 \mathrm{~s}, 0.04 \mathrm{~s}$ and $0.06 \mathrm{~s}$ after impact for the $15^{\circ} \mathrm{C}$ and $50^{\circ} \mathrm{C}$ films using droplets with Weber numbers 80,160 and 240

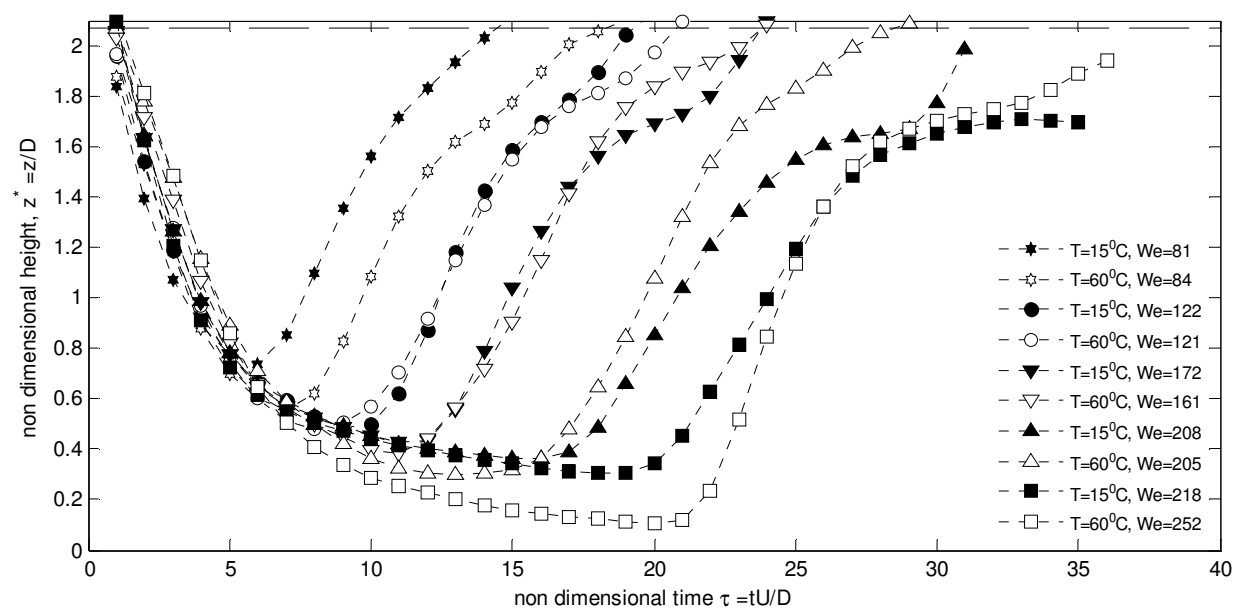

Figure 3. The effect of increasing the film temperature on the depth of the crater and its evolution with time. 


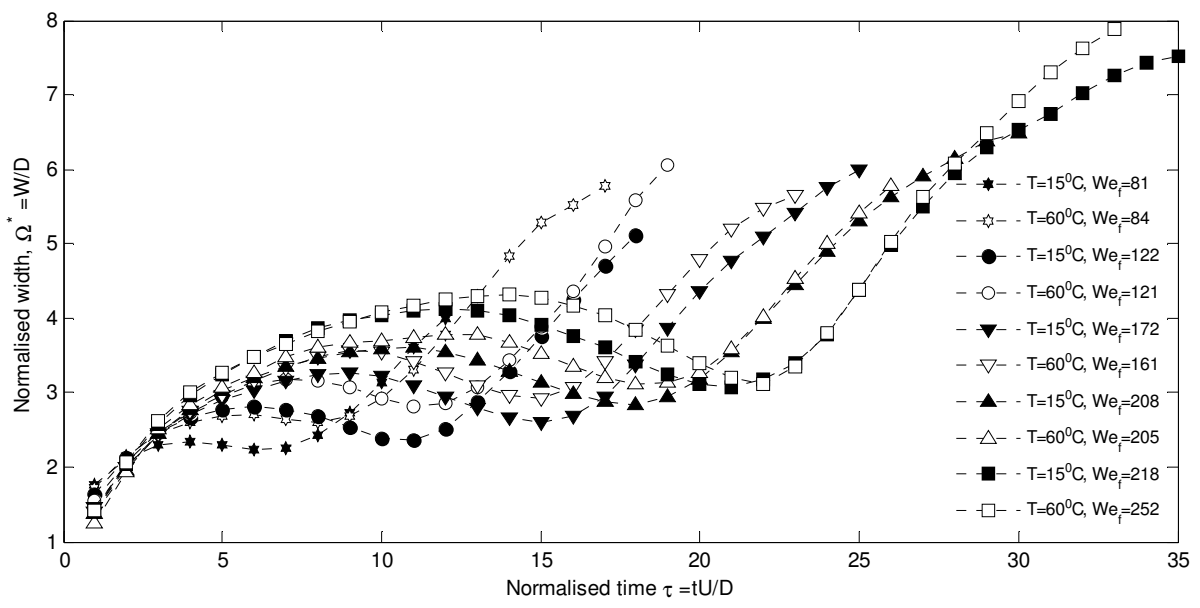

Figure 4. The effect of film temperature on the crater width and evolution of the crater with time.

\section{Conclusions and discussion}

The experiment outlined in this paper has demonstrated that changing the film temperature has an effect on the droplet impact outcome. Video evidence shows that secondary droplets are more frequent, but smaller if the temperature is increased. Three regimes can be identified in the impact; the crater expansion, crater slowing and crater collapse. Film temperature is shown to have little effect in the initial stages of the droplet impact. In this region, more commonly called the "inertial self-similar" region, the impact is dominated by momentum exchange [4], and the track of the crater evolution follows a similar pattern for all impact velocities and film temperatures. In the second region, the crater becomes deeper and wider as the temperature increase. In the collapsing regime, the crater becomes self-similar again for constant Webber number. These results will be used to understand in better detail the effect of cold droplets impacting on a hotter film.

\section{References}

1. Worthington, A.M., Nature,. 16, 165-166 (1877).

2. Rein, M., Fluid Dynamics Research, 12(2), 61 (1993).

3. Yarin, A.L., Annual Review of Fluid Mechanics. 159 (2006).

4. Bisighini, A., et al., Physical Review E - Statistical, Nonlinear, and Soft Matter Physics, 82 2010.

5. Hann, D.B., et al., Experiments in Fluids, 57, 1-12 (2016).

6. $\quad$ Manzello, S.L. and J.C. Yang, Experiments in Fluids, 32, 580-589 (2002).

7. Manzello, S.L. and J.C. Yang, Physics of Fluids, 15, 257-260 (2003).

8. Berberović, E., et al., Physical Review E - Statistical, Nonlinear, and Soft Matter Physics, 79, 2009. 